\title{
An Appraisal Of Food Serving Characteristics Among Patients With Type 2 Diabetes Mellitus Attending A Tertiary Care Diabetes Facility In Sri Lanka
}

\section{Abstract}

Introduction: The primary objective of the present study was to identify the portion sizes of food consumed by adult type 2 diabetic patients in Sri Lanka and to evaluate if these were within the current recommendations.

Materials and Methods: A cross sectional survey was performed at a tertiary care diabetes facility in Sri Lanka.

Starch, fruits, vegetables, meat or alternative, pulses, dairy and added sugar consumption was assessed according to standard methods using a 24-hour dietary recall.

Results: One hundred and twenty three randomly selected adult type 2 diabetic patients were studied. Forty six percent of participants exceeded the national recommendation of 4.5 to 9 servings of starch per day for patients with diabetes. Mean daily consumption of starch was 10.5 (median 10.2 IQR 4.7) servings. Only $26 \%$ and $45 \%$ of participants consumed the recommended 2 servings of fruits and 3 servings of vegetables per day. Thirty three percent and $8 \%$ did not consume any fruits or vegetables respectively. Dairy products were consumed by $25 \%$ only. Added sugar was not used by $99 \%$ of participants. Portion size was smaller in females and with advancing age. The mean proportions of total Carbohydrates, proteins and fats comprising total energy intake were $68.1 \%, 11.5 \%$ and $20.2 \%$ respectively.

Discussion: Medical nutrition therapy in diabetes recommends a mixed diet with carbohydrates from a variety of sources such as cereals, fruits and vegetables. Although Sri Lankan patients with diabetes consume less starch and more fruits and vegetables compared to non-diabetic counterparts, a substantial proportion still fail to meet the recommendations.
Arjuna Medagama ${ }^{1}$ Heshan Widanapathirana²

1 Department of Medicine, Faculty of Medicine, University of Peradeniya, Sri Lanka.

2 Department of Medicine.

University of Peradeniya, Sri Lanka

Contact information:

Arjuna Medagama.

”arjuna.medagama@gmail.com

Keywords

Type 2 diabetes mellitus,

Dietary survey, Portion sizes 


\section{Background}

Sri Lanka is a developing country with a population of 21 million and a rapidly increasing burden of diabetes. The prevalence had increased from $2.5 \%$ in 1990 to $10.3 \%$ in 2008, with a projected prevalence of $13.9 \%$ for the year 2025.[1, 2] The increase in prevalence is seen in both rural and urban areas [1, 2]. Sri Lanka has a high burden of non-communicable diseases (NCD), and in 2005 the prevalence of hypertension, diabetes and dysglycaemia was $20 \%$, $14 \%$ and $20 \%$ respectively [3]. The mortality from cardiovascular disease is recognized to be one of the highest worldwide. Ischemic heart disease (IHD) is the leading cause of death in Sri Lanka and accounts for 27.6 deaths per 100,000 people. Data from 2004 to 2012 show a steady increase in hospital mortality from IHD; in 2012 it accounted for $14.4 \%$ all hospital deaths [4].

The exact reason for this high mortality is unknown but is probably contributed to by the clustering of cardiovascular risk factors. This epidemic of NCDs is most likely associated with unhealthy eating patterns of the community[5].

Rice is the principal staple of Sri Lankan diet and other carbohydrates are consumed in lesser quantities. A recent meta-analysis had evidence suggesting that higher consumption of white rice (with a high glycaemic index) may increase the incidence of diabetes in Asian populations. [6] Amarasekera et al reported the glycaemic control of diabetic patients in Sri Lanka to be suboptimal in 2014 [7], which is probably dependent on poor dietary practices.

The national [8] and international [9]recommendations advocate the consumption of 6-11 servings of carbohydrate per day for healthy individuals. However, a recent study by Jayawardena found an average starch consumption of 14.1 servings per day among healthy Sri Lankan adults [10]. Although there is limited data on the serving characteristics of healthy Sri Lankans, no data is available for the diabetic population.
An appraisal of the serving characteristics of type 2 diabetic patients would be important in understanding current dietary practices and planning future strategies to improve Medical Nutrition Therapy (MNT). The aim of this study was to find the average number servings of the principal food categories of starch, fruits, vegetables, pulses, meat or equivalent, dairy and added sugar consumed by a group of adult type 2 diabetic patients attending a tertiary care diabetes facility.

\section{Materials and Methods}

This study performed between May and August 2014, recruited one hundred and twenty eight randomly selected type 2 patients with diabetes, attending the diabetes facility at Teaching Hospital, Peradeniya, Sri Lanka. This diabetes facility caters to over 2000 type 2 diabetes patients from a multiethnic background and a sample size of 128 was deemed adequate for the population. Patients were recruited over 16 follow up clinic days by generating 8 random numbers for each clinic day. Five patients declined to participate.

Patients registered at this clinic comprise those who self refer themselves, those referred by the hospital outpatients department, general practitioners and by other regional hospitals. Thus the sample can be considered representative. All patients upon registration at the unit undergo at least one session of routine nutritional counseling by a trained nurse in diabetes.

Clinical criteria were used to determine whether a patient was type 2 or type 1 as the serological markers such as GAD antibodies are not routinely performed due their prohibitive cost.

Ethical review for the study was obtained from the Ethics review committee of the Faculty of Medicine, university of Peradeniya. Inclusion criteria included the presence of type 2 diabetes mellitus diagnosed according to prevailing diagnostic criteria; for a duration exceeding 6 months and having had routine 
formal nutrition counseling at the facility. Inability to give consent due to Physical, verbal or intellectual impairment was regarded as the exclusion criterion. The patients were briefed regarding the study and verbal consent was obtained and formally recorded.

\section{Data collection}

Two independent interviewers performed a 24-hour dietary recall on the recruited patients. The data was recorded onto a pre-printed format, which allowed either weight of the portion or size of the portion using the methods described below to be entered. The gathered data from the 2 interviewers were compared at the end of the day and if there was any discrepancy the patient was re-interviewed on another day. The patients were asked to recall what they ate during the preceding 24-hours in chronological order. The interviewers used common household utensils such as plates of varying sizes, bowls, cups, spoons and glasses to determine portion sizes. These were available in a variety of sizes for patients to go through and determine which best reflected their portion size. In instances that patients were able to recall weight of their portion, this was recorded. Common foods such as rice and curries were available within the interviewers room and the patients were asked to serve their usual portion size onto a plate and the weight of the portion was recorded. This was then compared with the portion size they had recalled using the common household utensils.

All the food recorded from the 24-hour dietary recall were categorized under seven categories: (i) cereals or equivalents (starchy food); (ii) vegetables; (iii) fruits; (iv) meat or alternatives; (v) pulses; (vi) diary; and (vii) added sugars.

\section{Portion size determination}

Data from the dietary recall was assigned to one of the seven categories mentioned above. In cases where the food were a mixture several food types, they were disaggregated and the component ingredients assigned to one of the seven categories mentioned above. Similar method was used in a previous survey on a similar cultural background [10]. The weight of the food in grams divided by the weight of one portion in grams; or the amount in household measures (cup, spoon) divided by amount of one portion depicted in household measure was used to determine the amount of portions consumed in each food category. The number of portions thus determined in each category was then totaled and divided by the number of participants to calculate the mean number of portions consumed for a specific category.

\section{Data analysis}

The Student t test was used to test for the differences in mean age, obesity indices and dietary factors. Median and interquartile range was used to assess the number of servings consumed per day for each food category. Linear regression was used to determine the associations between food portion size and demographic factors such as age, gender, level of education and BMI. The data analysis was conducted using the Statistical Package for the Social Sciences (SPSS, version 20) for Windows. A p value of 0.05 was used to determine any statistically significant difference.

\section{Results}

One hundred and twenty three patients with type 2 diabetes were enrolled in the study following informed consent. There were 43 (34.9\%) male and 80 (65.1\%) female participants. The mean age of the males and females were 57.8 and 55.2 years respectively. Ninety percent of the study population was Singhalese with remaining 10\% comprising Tamils and Muslims. Majority (77\%) had completed secondary education and $2.3 \%$ had completed tertiary education. The mean BMI of the males and females were $24.39 \mathrm{~kg} / \mathrm{m}^{2}$ and $25.2 \mathrm{~kg} / \mathrm{m}^{2}$ respectively. The socio-demographic profile of the study population is shown in Table 1. 
Table 1. Demographic characteristics and BMI of the sample of Diabetic patients.

\begin{tabular}{|l|c|c|}
\hline \multicolumn{1}{|c|}{ Variable } & Males $(\mathbf{n} \mathbf{4 3})$ & Females $(\mathbf{n = 8 0})$ \\
\hline Variable & Mean or $\%$ & Mean or $\%$ \\
\hline Ethnicity & & \\
\hline Sinhalese & $90.7 \%$ & $87.5 \%$ \\
\hline Muslim & $4.7 \%$ & $11.3 \%$ \\
\hline Tamil & $4.7 \%$ & $1.25 \%$ \\
\hline Education level & & \\
\hline No Schooling & $9.3 \%$ & $5 \%$ \\
\hline Primary & $11.6 \%$ & $15 \%$ \\
\hline Secondary & $76.7 \%$ & $77.5 \%$ \\
\hline Graduate & $2.3 \%$ & $2.5 \%$ \\
\hline \multicolumn{1}{|c|}{ BMI $\left(\mathrm{kg} / \mathrm{m}^{2}\right)$} & $24.4(\mathrm{SD} 3.9)$ & $25.1(\mathrm{SD} 4.0)$ \\
\hline
\end{tabular}

The estimated median daily portions of the seven food categories from the 24-hour dietary recall according to gender are tabulated in Table 2 .

On average, type 2 diabetes patients in our survey consumed 10.5 (median 10.2, IQR 4.7) servings of starch per day. Males and females consumed 11.8 (median 11.2, IQR 5.35) and 9.8 (median 9.6, IQR 4.5) servings respectively. The difference was statistically significant. $(P<0.05)$ Although there is no definite consensus on the quantity of recommended servings of starch for patients with diabetes, local and international bodies still lay out recommendations to help patients plan their daily meals. [11, 12]

Ninety eight percent of all diabetes patients (99\% of males and $96 \%$ of females) exceeded the recommended 4.5 servings of starch per day. Forty six percent $(53 \%$ of males and $38 \%$ of females) exceeded 9 servings per day, and a quarter of men consumed more than 14 servings of starch per day.

The mean daily consumption of fruits and vegetables were 1.3 (median 1, IQR 2.0) and 2.8 (median 2.6, IQR 2.0) servings respectively, without a significant difference between males and females. Only $26 \%$ of the participants ( $25 \%$ of males and $27 \%$ of females) consumed the minimum recommendation of 2 servings fruits per day and only $45 \%$ of the participants consumed the minimum recommendation of 3 servings of vegetables per day. At least $62 \%$ of the study population consumed the recommended 2 servings of meat or alternative while consumption of pulses meeting the recommended 2 servings was only in $28 \%$ of the population. No dairy products were consumed by $75 \%$ of the sample. Consumption of added sugars were minimal with a mean consumption of 0.1 servings per day.

Linear regression of the data showed significant decrease in portion size with the female gender $(P<0.001)$ and advancing age $(P<0.001)$. Although a trend towards smaller portion size was noted with

Table 2. Median and Inter-quartile ranges (IQR) of different food groups (portions/d) among the sample of diabetic patients.

\begin{tabular}{|l|c|c|c|c|c|c|}
\hline & \multicolumn{2}{|c|}{ All adults } & \multicolumn{3}{c|}{ Males } & \multicolumn{2}{c|}{ Females } \\
\hline \multicolumn{1}{|c|}{ Food group } & Median & IQR & Median & IQR & Median & IQR \\
\hline Starch & 10.2 & 4.7 & 11.3 & 5.4 & 9.7 & 4.5 \\
\hline Vegetables & 2.6 & 2.0 & 2.6 & 2.3 & 2.6 & 1.9 \\
\hline Fruits & 1.0 & 2.0 & 1.0 & 2.0 & 1.0 & 2.0 \\
\hline Meat or alternative & 1.0 & 1.5 & 1.0 & 2.0 & 1.0 & 1.5 \\
\hline Pulses & 0.6 & 1.3 & 1.0 & 1.7 & 0.3 & 1.1 \\
\hline Dairy products & 0.3 & 0.2 & 0.3 & 0.2 & 0.3 & 0.3 \\
\hline Added sugar & 0.0 & 0.0 & 0.0 & 0.0 & 0 & 0 \\
\hline
\end{tabular}


increasing levels of education., it failed to reach statistical significance $(P=0.6)$. Similarly, there was a trend towards larger portions with increasing BMI, however it too failed to reach statistical significance $(P=0.06)$.

In the current study, the mean proportions of total Carbohydrates, proteins and fats comprising total energy intake were $68.1 \%, 11.5 \%$ and $20.2 \%$ respectively.

\section{Discussion}

Sri Lanka is a country with a rapidly expanding population of diabetic patients and its prevalence has grown exponentially over the last 2 decades [2]. At present one in five adults are known to have either diabetes or impaired glucose tolerance [1].

We performed this study among type 2 diabetes patients attending a tertiary care facility to find out the serving characteristics among them. Serving characteristics of diabetic patients have not been reported before in Sri Lanka and we believe it lays down the foundation for a larger study.

The participants of this study were from semiurban and rural backgrounds. The mean age of the participants was 56.5 years and the majority was female (61.5\%). Most of the participants (77\%) had completed secondary education.

Previous meta-analysis suggest a link between the consumption of white rice and the incidence of type 2 diabetes mellitus [6]. Sri Lanka records growing per capita rice consumption from $73 \mathrm{~kg}$ in 1973 to $110 \mathrm{Kg}$ in 2008 . Ninety percent of the rice consumed locally is white [14]. A recent study by Jayawardena et al reported a very high consumption of starch among healthy Sri Lankan adults [10]. In this study, $70 \%$ of the study population exceeded the recommended number of starch servings for a day for healthy adults. In addition, the same study reported a daily fruit and vegetable consumption amounting to only 0.43 and 1.73 servings respectively. These were well below the recommended daily fruit and vegetable consumption of 2 and 3 servings according to national and international guidelines $[8,9]$. The added sugar use was very high, amounting to 3.56 servings per day. The method used for portion size estimation in this study was similar to the current study making it appropriate to compare the data.

The endocrine society of Sri Lanka in their guidelines recommend 4.5 servings of starch for diabetic adults intending to lose weight and 9 servings of starch for those aiming at weight maintenance [11]. Similarly, 6-10 servings of starch is recommended for patients with diabetes in the United States [15] and 6-11 servings is recommended in the UK [12]. The lesser number of servings recommended for Sri Lankan diabetic patients is in keeping with the smaller build of the population.

Food intake is generally stated in grams of food consumed but recommendations are stated as numbers of servings such as cups, slices and pieces [13]. However these measures are often not standardized leading to greater ambiguity in quantifying food intake.

The average consumption of starch seems to be lower in diabetic individuals compared to healthy counterparts in Sri Lanka. Jayawardena et al in their study found a daily mean starch consumption of 14.06 servings, but the diabetic individuals of our study consumed only 10.5 servings on average. However this still remains well above the recommended intake and further counseling and other behavioral interventions may be necessary to curtail starch consumption. A comparison with the food intakes of the healthy Sri Lankan individuals is made to highlight the transition of food habits in diabetes; as traditionally Sri Lankans consume large portions of starch. We also highlight that there is a significant difference in the number of starch servings among diabetic males and females. The males consumed 11.8 servings daily compared 9.8 servings among females. $(P<0.05)$ This probably reflects the overall absolute low consumption of food by females. Moreover, the percentage of individuals who consu- 
med starch in excess of 14 servings per day had decreased from $41.6 \%$ from the national study to $13 \%$ in our study.

However, $98 \%$ of our study population still consumed more than 4.5 servings and $46 \%$ consumed more than 9 servings of starch per day, the lower and upper recommended levels of starch intake respectively $[8,11]$. The carbohydrate consumption amounted to $68.1 \%$ of the total daily energy intake. Fifty percent of carbohydrate was consumed as rice, while pulses, vegetables, wheat-based products and fruits contributed $44.7 \%$.

This high intake of carbohydrate is perhaps in keeping with the traditional serving habit of rice staple amounting to $3 / 4$ of the quantity of a meal. We highlight that this practice may have negative consequences on the control of diabetes in general and in particular on the post-prandial blood glucose. The American Diabetes Association (ADA) also emphasizes portion control for weight loss and maintenance in patients with diabetes.

At present there is not enough evidence to recommend one specific amount of carbohydrate to all people with diabetes. The relevance of low carbohydrate intake to good glycaemic control in diabetes is not clear. However, the emphasis should be on obtaining the carbohydrates from a variety of sources such as fresh fruits, vegetables and whole grain cereals and minimize same from sugars and fats [16].

Widely accepted dietary guidelines recommend at least 2 servings of fruits and 3 servings of vegetables daily for diabetic individuals $[12,15]$. The mean daily fruit and vegetable consumption in our study was 1.3 and 2.8 servings respectively and the combined fruit and vegetable consumption was 4.2 servings. The national survey among healthy Sri Lankans [10] found fruit and vegetable consumption to be 0.43 and 1.73 servings respectively with a combined consumption of 2.16 servings. We observed a considerable increase in the consumption of both fruits and vegetables in our study compa- red to non-diabetic Sri Lankans. However only $26 \%$ of the participants consumed the recommended 2 portions of fruits daily and $33 \%$ of did not consume fruits. More favourably, $46 \%$ consumed the recommended 3 servings of vegetables daily, and only $8 \%$ did not consume vegetables. This perhaps reflects a change in dietary practices as a result of health education as fruits and vegetables are relatively widely available in Sri Lanka.

The mean daily consumption of meats and pulses were 1.29 and 0.83 servings respectively with a combined consumption of 2.1servings. We observe a decrease in combined portion size of 2.78 from the national survey [10] to 2.1 in our study. However only $11 \%$ of both males and females consumed the recommended 3-4 servings of meat or alternative $[11,12,15]$ per day. We observed that proteins contributed $11.5 \%$ of the total daily energy intake of diabetes compared against the recommended contribution of $10-20 \%$ [17-19].

Although pulses are categorized under protein sources, the main nutrient in pulses would be carbohydrate. Therefore the mean daily consumption of 0.83 servings of pulses actually adds to the carbohydrate intake. Data available from previous studies is insufficient to make a recommendation on the amount of protein intake in diabetes. [16]

Dairy products are considered a key element in a diet and a rich source of calcium, vitamins and proteins. The mean daily consumption of dairy products was 0.36 servings and $75 \%$ of participants did not use dairy products. This is well below the recommended 1 to 3 servings per day for diabetic individuals [12, 15]. A comparison of Sri Lankan, UK and American recommendations for food consumption for diabetic individuals against the average portion sizes of the food consumed in the present study is given in table 3 .

We found a mean BMl of $24.4 \mathrm{~kg} / \mathrm{m}^{2}$ (SD 3.8) and $25.1 \mathrm{~kg} / \mathrm{m}^{2}$ (SD3.9) among male and female participants respectively. Katulanda et al also reported a mean BMI of $23.8 \mathrm{~kg} / \mathrm{m}^{2}(+-4.06)$ in dia- 
Table 3. Comparison of the average food intake of Diabetic patients with national and international recommendations.

\begin{tabular}{|l|c|c|c|c|}
\hline \multicolumn{1}{|c|}{ Food group } & $\begin{array}{c}\text { US recommendations } \\
\text { for diabetic patients } \\
\text { (Servings/d) [15] }\end{array}$ & $\begin{array}{c}\text { UK recommendations } \\
\text { for diabetic patients } \\
\text { (Servings/d) 12] }\end{array}$ & $\begin{array}{c}\text { Sri Lankan } \\
\text { Median intakes in } \\
\text { recommendations for } \\
\text { diabetic Adults [8, 11] } \\
\text { (Servings/d) }\end{array}$ & $\begin{array}{c}\text { Matients with diabetes } \\
\text { (Servings/d) }\end{array}$ \\
\hline Starch & $6-10$ & $6-11$ & $4.5-9$ & 10.2 \\
\hline Fruits & $2-4$ & $2-4$ & $2-3$ & 1.0 \\
\hline Vegetables & $3-4$ & $3-5$ & $3-5$ & 2.6 \\
\hline Meat and pulses & $4-7$ & $4-6$ & 3 & 1.0 \\
\hline Dairy & 2 & $2-3$ & $1-3$ & 0.3 \\
\hline Added sugars & Limit & Never/rare & - & 0.0 \\
\hline
\end{tabular}

betic patients and BMl of $21.7 \mathrm{~kg} / \mathrm{m}^{2}$ in healthy adults in 2 nationwide surveys [1, 20]. The overall prevalence of over-weight and diabetes among Sri Lankans is $25.8 \%$ and $9.2 \%$ respectively using the Asian cut off values [20]. We observed a much higher prevalence of $43 \%$ and $23 \%$ of over-weight and obesity among our participants. Horikawa et al reported mean BMI of $22.9 \mathrm{~kg} / \mathrm{m}^{2}$ in Japanese diabetic patients [21] and the mean BMI was much higher in western diabetic patients [22]. The higher percentage of over-weight and obese patients in our study probably reflects poor dietary practices in the adult population in general and the subsequent higher incidence of diabetes in the community. It also emphasizes the need for better health education measures.

We clarified that our study patients consumed a high-carbohydrate, low-fat diet. The use of fruits, vegetables and meat or alterative was adequate but use of dairy products was minimal. Consumption of starches was significantly higher in males, but the consumption of the remaining 6 food categories was similar among the males and females. We note a positive trend towards smaller starch portions and an almost complete abstinence from added sugar in the present study. The current sample of patients consumed more fruits vegetables compared to healthy adults.
Linear regression of the data showed a statistically significant decrease of portion size with female gender and advancing age. A non-significant increase of portion size was noted with increasing BMI. The portion sizes of carbohydrates were smaller in those with secondary and tertiary education. Generally the energy intakes and portions sizes of males are higher compared to females $[23,24]$. In developed countries energy intake is inversely associated with education levels [25]. In Sri Lanka too, Jayawardena et al demonstrated that energy intake is less in those with university education [24]. Although a non-significant tendency towards larger portion sizes was seen with rising BMI, there are other studies that demonstrated portion size is not associated with BMI [26]. Similarly, Horikawa et al demonstrated that energy intake among adult diabetic Japanese patients was not dependent on age or sex [21]. However, this study was not adequately powered to detect these associations and future studies should assess the associations between portion size, energy intake and demographic and diabetes related variables.

\section{Limitations}

Limitations of this study include the relatively small sample size of 123 patients from a single site and the use of a single 24-hour recall to assess the die- 
tary habits. The inclusion of patients with advancing age may have had an adverse effect on recall. A selection bias may have been introduced in the selection process due to exclusion of patients who were unable to give consent. However, we believe this data is valuable as none is available at present, and large-scale studies would help to define these parameters better. This study was confined to the estimation serving sizes of the seven food categories among type 2 diabetic individuals. Further analysis of the data to characterize the energy intakes, contribution of carbohydrates, proteins and fat to the daily energy consumption in detail would help to characterize the food habits better.

\section{Conclusion}

Medical nutrition therapy in diabetes recommends a mixed diet and carbohydrates from a variety of sources such as cereals, fruits and vegetables. Portion restriction in diabetes is recommended for those who are obese or over weight. In the current sample, majority of diabetic patients still consume more than the recommended amount of starch servings per day. Expression of portion size as multiples of servings is a practical way of translating medical nutrition therapy to everyday eating habits of patients, as a large portion of the study sample failed to meet the dietary recommendations, a larger nationwide survey is strongly recommended to assess these practices in detail.

\section{Acknowledgements}

The authors declare that there is no conflict of interest. This research received no specific grant from any funding agency, commercial or not-for-profit sectors

\section{References}

1. Katulanda P, Constantine GR, Mahesh JG, Sheriff R, Seneviratne $\mathrm{RD}$, Wijeratne $\mathrm{S}$, et al. Prevalence and projections of diabetes and pre-diabetes in adults in Sri Lanka-Sri Lanka Diabetes, Cardiovascular Study (SLDCS). Diabetic medicine: a journal of the British Diabetic Association. 2008; 25(9): 1062-9.

2. Illangasekera U, Rambodagalla S, Tennakoon S. Temporal trends in the prevalence of diabetes mellitus in a rural community in Sri Lanka. The journal of the Royal Society for the Promotion of Health. 2004; 124(2): 92-4.

3. Wijewardene K, Mohideen MR, Mendis S, Fernando DS, Kulathilaka T, Weerasekara D, et al. Prevalence of hypertension, diabetes and obesity: baseline findings of a population based survey in four provinces in Sri Lanka. The Ceylon medical journal. 2005; 50(2): 62-70.

4. Medical Statistics Unit Ministry of Health. Annual Health Bulletin 2012. Colombo: Ministry of Health Sri Lanka; 2012.

5. Abeywardena MY. Dietary fats, carbohydrates and vascular disease: Sri Lankan perspectives. Atherosclerosis. 2003; 171(2): 157-61.

6. Hu EA, Pan A, Malik V, Sun Q. White rice consumption and risk of type 2 diabetes: meta-analysis and systematic review. BMJ. 2012; 344: e1454.

7. Amarasekara AA FW, Wimalasekera SW,Turale S, Chanprasit S. Cross-sectional study of glycemic control among adults with type 2 diabetes. Nurs Health sci. 2014.

8. Nutrition Division Ministry of Health. Food based dietary guidelines for Sri lanka, 2nd Ed, colombo: Nutrttion division Minsitry of Health, Colombo. 2011.

9. US Department of Agriculture UDo. The food guide pyramid. Home and garden bulletin no 252. Washington DC: USDA Centre for Nutrition Policy and Promotion 1992.

10. Jayawardena R, Byrne NM, Soares MJ, Katulanda P, Hills AP. Food consumption of Sri Lankan adults: an appraisal of serving characteristics. Public health nutrition. 2013; 16(4): 653-8.

11. Somasundaram NP WC, De Silva S, Siribaddana S Clinical guidelins: The endocrine society od Sri Lanka. Sri Lanka Journal of Diabetes, endocrinology and Metabolism. 2013(3): 45-57.

12. Diabetes.co.uk. The Diabetes Food Pyramid 2015. Available from: http://www.diabetes.co.uk/food/diabetes-food-pyramid. html.

13. Cleveland LE, Cook DA, Krebs-Smith SM, Friday J. Method for assessing food intakes in terms of servings based on food guidance. Am J Clin Nutr. 1997; 65(4 Suppl): 1254S-63S.

14. D.P.C. Swarnasiri PJS, editor Quality evaluation of localy available rice in local market. Institiute of post harvest technology Sri Lanka symposium 2012; 2012; Anuradhapura Sri Lanka.

15. US Department of Health and Human Services. Eating and Diabetes 2014. Available from: http://diabetes.niddk.nih.gov/ DM/PUBS/eating_ez/index.aspx. 
16. Evert AB, Boucher JL, Cypress M, Dunbar SA, Franz MJ, MayerDavis EJ, et al. Nutrition therapy recommendations for the management of adults with diabetes. Diabetes care. 2013; 36(11): 3821-42.

17. AACE Diabetes Mellitus Clinical Practice Task force, AACE medical guidelines for the clinical practice for the management of diabetes mellitus. Endocr Pract. 2007; 13: 1-27.

18. Mann JI, De Leeuw I, Hermansen K, Karamanos B, Karlstrom B, Katsilambros N, et al. Evidence-based nutritional approaches to the treatment and prevention of diabetes mellitus. Nutrition, metabolism, and cardiovascular diseases: NMCD. 2004; 14(6): 373-94.

19. Canadian Diabetes Association Canadian Diabetes Association 2008 Clinical Practice Guidelines for the Prevention and Management of Diabetes in Canada. Can J Diabetes. 2008; 32: S40-6.

20. Katulanda $P$, Jayawardena MA, Sheriff MH, Constantine GR, Matthews DR. Prevalence of overweight and obesity in Sri Lankan adults. Obesity reviews: an official journal of the International Association for the Study of Obesity. 2010; 11(11): 751-6.

21. Horikawa C, Yoshimura Y, Kamada C, Tanaka S, Tanaka S, Takahashi $A$, et al. Dietary intake in Japanese patients with type 2 diabetes: Analysis from Japan Diabetes Complications Study. Journal of diabetes investigation. 2014; 5(2): 176-87.

22. Ma $Y$, Olendzki $B C$, Hafner $A R$, Chiriboga $D E$, Culver $A L$, Andersen $V A$, et al. Low-carbohydrate and high-fat intake among adult patients with poorly controlled type 2 diabetes mellitus. Nutrition. 2006; 22(11-12): 1129-36.

23. Caster W. Systemtaic estimation of food intakes from food frequency data. Nutr Res. 1986; 6: 469-72.

24. Jayawardena R, Thennakoon S, Byrne N, Soares M, Katulanda $P$, Hills A. Energy and nutrient intakes among Sri Lankan adults. International archives of medicine. 2014; 7: 34.

25. Rennie KL, Jebb SA. Prevalence of obesity in Great Britain. Obesity reviews: an official journal of the International Association for the Study of Obesity. 2005; 6(1): 11-2.

26. Livingstone MB, Pourshahidi LK. Portion size and obesity. Advances in nutrition. 2014; 5(6): 829-34.

\section{Comment on this article:}

\section{(f) 8 in $8+\boldsymbol{S} P$}

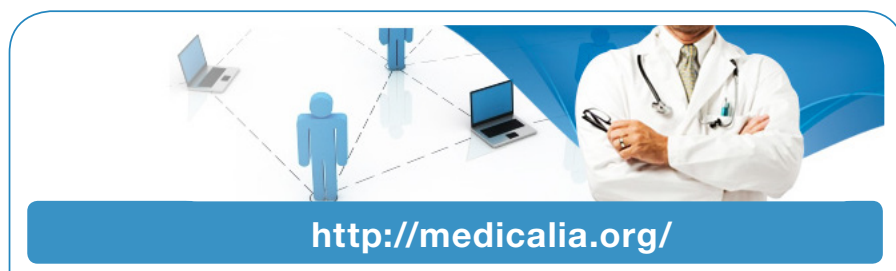

Where Doctors exchange clinical experiences, review their cases and share clinical knowledge. You can also access lots of medical publications for free. Join Now!

\section{Publish with iMedPub}

\section{http://www.imed.pub}

International Archives of Medicine is an open access journal publishing articles encompassing all aspects of medical science and clinical practice. IAM is considered a megajournal with independent sections on all areas of medicine. IAM is a really international journal with authors and board members from all around the world. The journal is widely indexed and classified Q1 in category Medicine. 